\title{
Set-invariance characterizations of discrete-time descriptor systems with application to active mode detection ${ }^{\sqrt{4}}$
}

\author{
Ye Wang a,b,*, Sorin Olaru c, Giorgio Valmorbida c, Vicenç Puig b, Gabriela Cembrano b,d \\ a College of Automation, Harbin Engineering University, No. 145 Nantong Street, Nangang District, 150001 Harbin, P.R. China \\ ${ }^{\mathrm{b}}$ Advanced Control Systems (SAC) Research Group at Institut de Robòtica i Informàtica Industrial (IRI), CSIC-UPC, Universitat Politècnica de \\ Catalunya-BarcelonaTech (UPC), C/. Llorens i Artigas 4-6, 08028 Barcelona, Spain \\ ${ }^{\mathrm{c}}$ Laboratoire des Signaux et Systèmes, CentraleSupélec, CNRS, Univ. Paris-Sud, Université Paris-Saclay, 3 Rue \\ Joliot-Curie, 91192 Gif-sur-Yvette, France \\ d Cetaqua, Water Technology Centre, Ctra. d'Esplugues 75, Cornellà de Llobregat, 08940 Barcelona, Spain
}

\begin{abstract}
A B S T R A C T
In this paper, we study robust invariant set characterizations of discrete-time descriptor systems and propose an active mode detection mechanism for discrete-time descriptor systems considering multiple modes of operation. The considered class of descriptor systems assumes regularity, stability and is affected by unknown-but-bounded disturbances. As a first theoretical result, we establish a general framework for robust invariant sets for discrete-time descriptor systems in both causal and non-causal cases. Particular transformations are subsequently proposed for handling causal and noncausal descriptor systems and will be used to characterize the effects of disturbances. Based on these set-theoretic notions and a designed input signal for active set separations, we propose an active mode detection mechanism by exploiting the strong invariance properties.
\end{abstract}

\section{Introduction}

Set-invariance theory has played an essential role in automatic control with a variety of applications to control systems, since it is widely used for guaranteeing the stability and achieving desired performance (Blanchini, 1999; Kolmanovsky \& Gilbert, 1998). For systems affected by disturbances, different techniques in set-invariance theory are used for the computation of robust invariant (RI) sets. These techniques have been applied to linear dynamical systems (Raković, Kerrigan, Kouramas, \& Mayne, 2005), linear parameter-varying systems (Seron \& De Doná, 2015), and nonlinear systems (Alamo, Cepeda, Fiacchini, \& Camacho, 2009). In particular, ultimate boundedness methods are used to compute RI sets with relative low complexity (Kofman, Haimovich, \& Seron, 2007; Olaru, De Doná, Seron, \& Stoican, 2010). Furthermore, set-invariance characterizations are instrumental for

\footnotetext{
the material in this paper was partially presented at the 56th IEEE Conference on Decision and Control, December 12-15, 2017, Melbourne, Australia. This paper was recommended for publication in revised form by Associate Editor Denis Arzelier under the direction of Editor Richard Middleton.

* Corresponding author at: College of Automation, Harbin Engineering University, No. 145 Nantong Street, Nangang District, 150001 Harbin, P.R. China.

E-mail addresses: yewang@hrbeu.edu.cn (Y. Wang), sorin.olaru@12s.centralesupelec.fr (S. Olaru), giorgio.valmorbida@12s.centralesupelec.fr (G. Valmorbida), vicenc.puig@upc.edu (V. Puig), gabriela.cembrano@upc.edu (G. Cembrano).
}

control strategies, such as fault detection and isolation (Blanchini, Casagrande, Giordano, Miani, Olaru, \& Reppa, 2017; Xu, Puig, Ocampo-Martinez, Stoican, \& Olaru, 2014), fault-tolerant control (Olaru et al., 2010; Seron, De Doná, \& Olaru, 2012; Stoican \& Olaru, 2013) and robust model predictive control (Mayne, Seron, \& Raković, 2005). A remarkable application of RI sets is on mode detection of systems subject to multiple modes of operation. Indeed, since different operating modes lead to different RI sets, the distance between these sets can be used for monitoring and mode detection. Due to the fact that the RI sets of different modes may overlap, an additive input signal can be conveniently designed to separate a parametrization of the RI sets, represented by tubes of trajectories. In this case, the set-theoretic mode detection mechanism is called active. In the literature, this mechanism is also called active fault diagnosis (Raimondo, Marseglia, Braatz, \& Scott, 2016). A set of additive inputs are designed to guarantee fault diagnosis outputs that are only consistent with one faulty scenario. These additive inputs can be obtained from the solution to a mixed-integer quadratic program or using a multi-parametric approach (Marseglia \& Raimondo, 2017).

The above described methods have been proposed for standard dynamical systems modeled only with differential/ difference equations. Due to mass, volume or energy conservation laws, difference equations describing a dynamical system can be coupled with a set of algebraic equations. This class of systems described by differential/difference and algebraic equations is 
called descriptor, singular, or differential/difference-algebraic systems (Dai, 1989; Duan, 2010). For this class of systems, setinvariance characterizations as well as their mode detection are still not widely developed. Instances of such systems can be found in water distribution networks (Wang, Puig \& Cembrano, 2017), chemical processes (Biegler, Campbell, \& Mehrmann, 2012), electrical circuits (Riaza, 2008) and economic models (Dai, 1989). From a theoretical point of view, descriptor systems satisfying a well-posed property, for which a solution exists and is unique, are called regular (Dai, 1989; Oară \& Andrei, 2013). Regularity, however, does not imply causality and models of interest in economy are non-causal, see e.g. the Leontief model (Dai, 1989). In terms of a control system, stability (Halanay \& Rasvan, 2000) is an important property for the analysis of boundedness and convergence of the closed-loop trajectory. In particular, in terms of descriptor systems, admissibility guarantees the properties of regularity, causality and stability. The present paper aims to revisit all these properties of descriptor systems and exploit the underlying structural properties in a set-theoretic framework.

Systems modeled in a descriptor framework can be affected by uncertainties, such as modeling errors and disturbances. Also, faults from actuator and sensor malfunctions may change the dynamics and constraints of the system and therefore the system can evolve or switch to different modes of operation. For instance, in cyber-physical systems, the system model can be changed by faults of different nature, such as process/system faults, actuator and sensor faults, as well as communication faults. Thus, a suitable mode detection mechanism is required to identify whether the actual cyber-physical system matches with the prediction by checking the feedback information.

The main contribution of this paper is to present a general framework for set-invariance characterization of discrete-time descriptor systems as well as an application to active mode detection. The proposed computation of invariant sets relies on partitioning the state space for both causal and non-causal descriptor systems under standard notations. Besides, we propose an active mode detection mechanism based on positive set-invariance characterizations for discrete-time descriptor systems.

The preliminary results presented in this paper have been reported in Wang, Olaru, Valmorbida, Puig and Cembrano (2017). Additional improvements and new contribution are summarized as follows:

- (A detailed review of definitions and properties of discrete-time descriptor systems) These properties prove to be useful for the computation of RI sets.

- (A general framework for set-invariance characterizations of discrete-time descriptor systems) This framework completes the preliminary results in Wang, Olaru et al. (2017). In addition, the convergence time for each RI set and the results of checking the compatible initial states are provided.

- (A novel active mode detection mechanism) The strong invariance properties for detecting mode of operation are formulated.

\section{Background and preliminaries}

\subsection{Discrete-time descriptor systems}

Consider the discrete-time linear time-invariant (LTI) descriptor system with additive disturbances

$E x(k+1)=A x(k)+B_{w} w(k)$,

where $x \in \mathbb{R}^{n}$ and $w \in \mathbb{R}^{q}$ denote the state vector and the disturbance vector, respectively, $k \in \mathbb{N}$. $A \in \mathbb{R}^{n \times n}, B_{w} \in \mathbb{R}^{n \times q}$ and $E \in \mathbb{R}^{n \times n}$ with $\operatorname{rank}(E)=r \leq n$.
Definition 1 (Regularity). A descriptor system (1) is said to be regular if it has a unique solution defined as an application $x$ : $\mathbb{N} \rightarrow \mathbb{R}^{n}$ which satisfies (1) for any disturbance realization $w$ : $\mathbb{N} \rightarrow \mathbb{R}^{q}$ and a compatible initial state $x(0)$.

From the above definition, if the system (1) is regular, then it has a unique solution for the disturbance-free case $(w \equiv 0)$. We also say the matrix pair $(E, A)$ is regular.

Definition 2 (Causality). A regular descriptor system (1) is said to be causal if $x(k), \forall k \in \mathbb{N}$ is determined completely by the initial condition $x(0)$ and $w(j)$, for $j=0, \ldots, k$. Otherwise, it is said to be non-causal.

Definition 3 (Asymptotic Stability). A regular descriptor system (1) is said to be asymptotically stable for the disturbances-free case $(w \equiv 0)$ if $\lim _{k \rightarrow \infty} x(k)=0$.

Definition 4 (Admissibility). A descriptor system (1) for the disturbances-free case $(w \equiv 0)$ is said to be admissible if it is regular, causal and asymptotically stable.

Lemma 1 (Dai, 1989). For the matrix pair $(E, A)$ of the descriptor system (1), the following properties hold

- (Regularity) the pair $(E, A)$ is regular if $\operatorname{det}(z E-A)$ is not identically zero.

- (Causality) the pair $(E, A)$ is causal if $\operatorname{deg}(\operatorname{det}(z E-A))=$ $\operatorname{rank}(E)$.

- (Asymptotic stability) the pair $(E, A)$ is asymptotically stable if $|v|<1, \forall v \in \lambda(E, A)$, where $\lambda(E, A)$ denotes the generalized eigenvalues of $E$ and $A$.

In the following, admissibility is not part of the assumption, i.e. the study concerns both causal and non-causal descriptor systems.

Assumption 1. The descriptor system (1) (the matrix pair $(E, A)$ ) is regular and asymptotically stable in the disturbance-free case $(w \equiv 0)$.

We now establish suitable transformations that decompose the descriptor system (1) into subsystems for set-invariance characterizations and active mode detection.

Definition 5 (Equivalence of Descriptor Systems). Consider two descriptor systems respectively defined by the triplets $\left(E, A, B_{w}\right)$ and $\left(\tilde{E}, \tilde{A}, \tilde{B}_{w}\right)$. If there exists a pair of non-singular matrices $Q \in$ $\mathbb{R}^{n \times n}$ and $P \in \mathbb{R}^{n \times n}$ satisfying $Q E P=\tilde{E}, Q A P=\tilde{A}, Q B_{w}=\tilde{B}_{w}$, then these two systems are called restricted equivalent under the transformation $(Q, P)$.

For the descriptor system (1), we now present two standard restricted equivalent forms that are of interest (Duan, 2010, Chapter 2).

Consider the descriptor system (1) with $\operatorname{rank}(E)=r$. There always exists a transformation $(Q, P)$ yielding

$Q E P=\left[\begin{array}{cc}I_{r} & 0 \\ 0 & 0\end{array}\right], Q A P=\left[\begin{array}{ll}A_{1} & A_{2} \\ A_{3} & A_{4}\end{array}\right], Q B_{w}=\left[\begin{array}{l}B_{w 1} \\ B_{w 2}\end{array}\right]$,

with $A_{1} \in \mathbb{R}^{r \times r}, A_{2} \in \mathbb{R}^{r \times(n-r)}, A_{3} \in \mathbb{R}^{(n-r) \times r}, A_{4} \in \mathbb{R}^{(n-r) \times(n-r)}$, $B_{w 1} \in \mathbb{R}^{r \times q}$ and $B_{w 2} \in \mathbb{R}^{(n-r) \times q}$.

Lemma 2 (Dynamics Decomposition Form (Duan, 2010)). The descriptor system (1) is causal if and only if there exists a transformation $(Q, P)$ yielding (2) with a non-singular block matrix $A_{4}$. 
Based on the above lemma, an equivalent causal descriptor system in a standard dynamical form is presented in the following.

Lemma 3 (Equivalent Causal Descriptor System). A causal descriptor system (1) with $\operatorname{rank}(E)=r$ can be transformed into the following form

$\tilde{x}(k+1)=\tilde{A} \tilde{x}(k)+\tilde{B}_{w} \tilde{w}(k)$,

where

$$
\begin{aligned}
\tilde{A} & =\left[\begin{array}{cc}
A_{1}-A_{2} A_{4}^{-1} A_{3} & 0 \\
-A_{4}^{-1} A_{3}\left(A_{1}-A_{2} A_{4}^{-1} A_{3}\right) & 0
\end{array}\right], \\
\tilde{B}_{w} & =\left[\begin{array}{cc}
B_{w 1}-A_{2} A_{4}^{-1} B_{w 2} & 0 \\
-A_{4}^{-1} A_{3}\left(B_{w 1}-A_{2} A_{4}^{-1} B_{w 2}\right) & -A_{4}^{-1} B_{w 2}
\end{array}\right] .
\end{aligned}
$$

and $A_{1}, A_{2}, A_{3}, A_{4}, B_{w 1}, B_{w 2}$ are defined in (2) and

$\tilde{x}(k)=\left[\begin{array}{c}\tilde{x}_{1}(k) \\ \tilde{x}_{2}(k)\end{array}\right]=P^{-1} x(k), \tilde{w}(k)=\left[\begin{array}{c}w(k) \\ w(k+1)\end{array}\right]$,

with $\tilde{x}_{1}(k) \in \mathbb{R}^{r}, \tilde{x}_{2}(k) \in \mathbb{R}^{(n-r)}$.

Proof. See the proof of Wang, Olaru et al. (2017, Lemma 3).

Remark 1. In Lemma 3, w(k+1) only appears in the algebraic equation of the descriptor system (1), which is used to compute $\tilde{x}_{2}(k+1)$.

The regular descriptor system (1) also allows the transformation in the so-called Kronecker canonical form.

Lemma 4 (Kronecker Canonical Form (Dai, 1989)). The descriptor system (1) is regular if and only if there exists a transformation $(\bar{Q}, \bar{P})$ yielding

$\bar{Q} E \bar{P}=\left[\begin{array}{cc}I_{p} & 0 \\ 0 & \bar{N}\end{array}\right], \bar{Q} A \bar{P}=\left[\begin{array}{cc}\bar{A} & 0 \\ 0 & I\end{array}\right], \bar{Q} B_{w}=\left[\begin{array}{l}\bar{B}_{w 1} \\ \bar{B}_{w 2}\end{array}\right]$,

with $\bar{A} \in \mathbb{R}^{p \times p}, \bar{B}_{w 1} \in \mathbb{R}^{p \times q}, \bar{B}_{w 2} \in \mathbb{R}^{(n-p) \times q}$. Moreover, $\bar{N} \in$ $\mathbb{R}^{(n-p) \times(n-p)}$ is a nilpotent matrix (that is there exists a scalar $s>0$ such that $\bar{N}^{s}=0$ and $\left.\bar{N}^{s-1} \neq 0, s \leq n-p\right)$ and $p \leq r=\operatorname{rank}(E)$.

Computationally efficient and numerically stable methods exist to obtain these transformations as reported in Gerdin (2004) and Varga (2017).

Lemma 5 (Causality (Dai, 1989)). The descriptor system (1) transformed in the Kronecker canonical form (6) is causal if and only if $\bar{N}=0$.

\subsection{Background of set-invariance theory}

We now introduce set-theoretic notions for discrete-time descriptor systems. For a regular and stable descriptor system (1), we consider that the additive disturbances are unknown but bounded in a known set

$w(k) \in \mathcal{W}=\left\{w \in \mathbb{R}^{q}:|w| \leq \bar{w}\right\}, \forall k \in \mathbb{N}$,

with $\bar{w} \in \mathbb{R}^{q}$ and elementwise inequality.

As a consequence of boundedness of the disturbances and the stability of the dynamics, the system trajectories eventually converge to a bounded region of the state space (Kolmanovsky \& Gilbert, 1998) for the forward trajectories. Given an initial state $x(0)$ and the unique solution to (1) (note that the discretetime domain of the solution may include negative values for backward propagations), the following definitions are introduced in terms of the set-theoretic analysis.
Definition 6 (RI Set). A set $\Omega \in \mathbb{R}^{n}$ is said to be robust invariant (RI) with respect to the system (1) if $x(0) \in \Omega$ implies $x(k) \in \Omega$, $\forall w(k) \in \mathcal{W}$ and $\forall k \in \mathbb{Z}$.

Definition 7 (RPI Set). A set $\Omega \in \mathbb{R}^{n}$ is said to be robust positively invariant (RPI) with respect to the system (1) if $x(0) \in \Omega$ implies $x(k) \in \Omega, \forall w(k) \in \mathcal{W}$ and $\forall k \in \mathbb{N}$.

Definition 8 ( $m R P I$ Set). An RPI set $\Omega_{\infty} \in \mathbb{R}^{n}$ is said to be minimal $\mathrm{RPI}(\mathrm{mRPI})$ with respect to the system (1) if it is contained in every closed RPI set.

Definition 9 (L-steps $R N I$ Set). A set $\Omega \in \mathbb{R}^{n}$ is $L$-steps robust negatively invariant (RNI) with respect to the system (1) if $x(L) \in$ $\Omega$ implies $x(L+k) \in \Omega, \forall w(k) \in \mathcal{W}$ and $\forall k \in \mathbb{Z}_{[-L, 0]}$.

\section{Robust invariant set characterizations of discrete-time de- scriptor systems}

In this section, we formulate explicit expressions of several RI sets and approximations of minimal RI sets for discrete-time descriptor systems in both causal and non-causal cases. Furthermore, the convergence time for each RI set is provided.

For an admissible descriptor system (1), the set analysis will be performed using the dynamics decomposition form. From Lemma 3, there exists a transformation $(Q, P)$ leading to (2) and (3)-(5). We consider a partition of the matrix $P$ as $P=$ $\left[P_{1}, P_{2}\right]$ with $P_{1} \in \mathbb{R}^{n \times r}$ and $P_{2} \in \mathbb{R}^{n \times(n-r)}$. The structure of the $\mathrm{mRPI}$ set of the admissible descriptor system (1) is characterized in the following theorem. ${ }^{1}$

Theorem 1 ( $m R P I$ Set of Admissible Descriptor Systems). Consider an admissible descriptor system (1) with the dynamics decomposition form in (2) and $w(k) \in \mathcal{W}, \forall k \in \mathbb{N}$. The mRPI set $\Omega^{c}$ is given by $\Omega^{c}=P_{1} \Phi_{1} \oplus P_{2} \Phi_{2}$, where

$\Phi_{1}=\bigoplus_{i=0}^{\infty} \tilde{A}_{1} \tilde{B}_{w 1} \mathcal{W}$

$\Phi_{2}=\left(-A_{4}^{-1} A_{3} \Phi_{1}\right) \oplus\left(-A_{4}^{-1} B_{w 2} \mathcal{W}\right)$,

with $\tilde{A}_{1}=A_{1}-A_{2} A_{4}^{-1} A_{3}$ and $\tilde{B}_{w 1}=B_{w 1}-A_{2} A_{4}^{-1} B_{w 2}$.

Proof. With the transformation $(Q, P)$, the descriptor system (1) is equivalent to a dynamical system including two subsystems as in (4). On the one hand, from (4) we have

$\tilde{x}_{1}(k+1)=\tilde{A}_{1} \tilde{x}_{1}(k)+\tilde{B}_{w 1} w(k)$.

The admissibility of (1) implies the matrix $\tilde{A}_{1}$ is Schur. Then, the characterization of the MRPI set for $\tilde{x}_{1}$ can be obtained as in (8a) using the standard LTI notions (Kolmanovsky \& Gilbert, 1998). On the other hand, from (2) we obtain $\tilde{x}_{2}(k)=-A_{4}^{-1} A_{3} \tilde{x}_{1}$ $(k)-A_{4}^{-1} B_{w 2} w(k)$, which is an algebraic equation. Thus, we obtain the mRPI set $\Phi_{2}$ by a linear projection image of the set $\Phi_{1}$ in (8a), which leads to (8b). By definition in (5) and using the Minkowski addition of the sets obtained via the linear mapping defined by the matrices $P_{1}$ and $P_{2}$, we can determine the mRPI set $\Omega^{c}=$ $P_{1} \Phi_{1} \oplus P_{2} \Phi_{2}$.

Remark 2. For invariant approximations of the mRPI set $\Omega^{c}$ in Theorem 1, several approaches can be applied to the LTI part of the dynamics leading to an approximation of $\Phi_{1}$ (see e.g. Olaru et al., 2010; Raković et al., 2005) while the approximation of $\Phi_{2}$ is a projection involving the approximation of $\Phi_{1}$ and $\mathcal{W}$.

1 The Minkowski sum is denoted by $\oplus$. 
Furthermore, by applying the iterative approximation approach in Olaru et al. (2010), arbitrarily close approximations can be obtained $\Omega_{0}^{c} \supseteq \cdots \supseteq \Omega^{c}$.

Based on the above results, we present a practical condition of the compatibility check for any initial state $x(0)$.

Corollary 1. Consider an initial state $x(0)$ for the admissible descriptor system (1) in (3)-(5). If

$\tilde{x}_{2}(0) \notin\left(-A_{4}^{-1} A_{3} \zeta^{*} \Phi_{1}\right) \oplus\left(-A_{4}^{-1} B_{w 2} \mathcal{W}\right)$,

where $x(0)=P_{1} \tilde{x}_{1}(0)+P_{2} \tilde{x}_{2}(0)$ and $\zeta^{*}=\min \left\{\zeta \in \mathbb{R}: \tilde{x}_{1}(0) \in\right.$ $\left.\zeta \Phi_{1}\right\}$, then $x(0)$ is not a compatible initial state for (1) and it is independent of any disturbance realization $w(0) \in \mathcal{W}$.

Proof. Based on the mRPI set $\Omega^{c}$, if $x(0)$ is compatible, then it holds $\tilde{x}_{2}(0) \in \Phi_{2}, \forall w(0) \in \mathcal{W}$. On the other hand, for a scalar $\zeta^{*}$, if the condition (10) does not hold, then $x(0)$ is not compatible. Note that the set in (10) is not an RPI set but it represents a constraint for the descriptor part of states whenever this constraint is violated, leading to the incompatibility of the algebraic equations.

Remark 3. By Definition 6 and its characterization in Theorem 1 , the consistency in terms of initial state $x(0)$ with the descriptor model (1) can be tested. In presence of the disturbance $w(0) \in \mathcal{W}$, $x(0)$ may not be a compatible initial state. This shows that $x(0)$ should be understood as an implicit function of $w(0)$, i.e. $x(w(0))$, by means of the solution of algebraic equations.

To complete the study of admissible descriptor systems, the computation result of the convergence time for discrete-time admissible descriptor systems is provided based on the result in Seron et al. (2012, Appendix A). This is equivalent to an upper bound for the total number of steps necessary for the system trajectories to reach the set $\Omega^{c}$ from a given initial state.

Theorem 2 (Convergence Time for Admissible Descriptor Systems). Consider an admissible descriptor system (1), w(k) $\in \mathcal{W}, \forall k \in \mathbb{N}$ and an approximation of $\Omega_{0}^{c} \supseteq \Omega^{c}$ with $\Omega_{0}^{c}=P_{1} \hat{\Phi}_{1,0} \oplus P_{2} \Phi_{2}$. For a compatible initial state $x(0)$, the system trajectory $x(k)$ belongs to $\Omega_{0}^{c}$, that is, $\tilde{x}_{1}(k)$ defined in (5) belongs to $\hat{\Phi}_{1,0}$, for $k \geq T_{c_{a}}$, where $T_{c_{a}}$ is the convergence time corresponding to (1) and depends on $x(0)$ and $\tilde{\varepsilon}$.

Proof. Based on Lemma 3, $\tilde{x}_{2}(k)$ has no dynamics and is a linear mapping of $\tilde{x}_{1}(k)$ and $w(k)$. By directly applying the result in Seron et al. (2012, Appendix A) to $\tilde{x}_{1}(k)$ with its dynamics $\tilde{x}_{1}(k+1)=$ $\tilde{A}_{1} \tilde{x}_{1}(k)+\tilde{B}_{w 1} w(k)$, we can obtain the convergence time $T_{c_{a}}$.

In case that the descriptor system (1) is regular and stable but not causal, there might exist a unique solution at each time (Dai, 1989). We now consider a non-causal and stable descriptor system (1) and use the Kronecker canonical form in (6) for the RPI characterization.

From Lemma 4, a non-causal descriptor system (1) can be transformed in (6) with a nilpotent matrix $\bar{N}$ satisfying $\bar{N} \neq 0$. As introduced in Dai (1989, Chapter 8), for a regular matrix pair $(E, A)$, there exists a suitable transformation $(\bar{Q}, \bar{P})$ with $\bar{P}=$ $\left[\begin{array}{ll}\bar{P}_{1} & \bar{P}_{2}\end{array}\right], \bar{P}_{1} \in \mathbb{R}^{n \times p}, \bar{P}_{2} \in \mathbb{R}^{n \times(n-p)}$ yielding to $(6)$.

For the transformed system in the Kronecker form, we use the following partitioning form

$\bar{x}(k)=\left[\begin{array}{l}\bar{x}_{1}(k) \\ \bar{x}_{2}(k)\end{array}\right]=\bar{P}^{-1} x(k), \bar{Q} B_{w}=\left[\begin{array}{l}\bar{B}_{w 1} \\ \bar{B}_{w 2}\end{array}\right]$,

with $\bar{x}_{1}(k) \in \mathbb{R}^{p}, \bar{x}_{2}(k) \in \mathbb{R}^{(n-p)}$.
Based on the Kronecker canonical form in Lemma 4, we have that

$$
\begin{aligned}
\bar{x}_{1}(k+1) & =\bar{A} \bar{x}_{1}(k)+\bar{B}_{w 1} w(k), \\
\bar{N} \bar{x}_{2}(k+1) & =\bar{x}_{2}(k)+\bar{B}_{w 2} w(k) .
\end{aligned}
$$

The structure in (12) highlights the fact that the non-causal descriptor system (1) is stable if and only if the matrix $\bar{A}$ is Schur. We now formulate the mRPI set of discrete-time non-causal descriptor systems.

Theorem 3 (mRPI Set of Non-causal Descriptor Systems). Consider a non-causal descriptor system (1) with the Kronecker canonical form in (6) and $w(k) \in \mathcal{W}, \forall k \in \mathbb{N}$. The mRPI set $\Omega^{n}$ is given by $\Omega^{n}=\bar{P}_{1} \Theta_{1} \oplus \bar{P}_{2} \Theta_{2}$, where

$$
\begin{aligned}
\Theta_{1} & =\bigoplus_{i=0}^{\infty} \bar{A}^{i} \bar{B}_{w 1} \mathcal{W}, \\
\Theta_{2} & =\bigoplus_{i=0}^{n-p-1}\left(-\bar{N}^{i} \bar{B}_{w 2} \mathcal{W}\right) .
\end{aligned}
$$

Proof. The non-causal descriptor system can be decomposed in two subsystems, where (12a) is an ordinary difference equation. Hence, the mRPI set of $\bar{x}_{1}$ can be constructed as in (13a). On the other hand, from (12b), the anti-causal state $\bar{x}_{2}(k)$ can be propagated as follows: $\bar{x}_{2}(k)=\bar{N} \bar{x}_{2}(k+1)-\bar{B}_{w 2} w(k), \bar{x}_{2}(k+1)=$ $\bar{N} \bar{x}_{2}(k+2)-\bar{B}_{w 2} w(k+1)$, and after the $(n-p)$-step iterations, we can obtain

$\bar{x}_{2}(k)=\bar{N}^{(n-p)} \bar{\chi}_{2}(k+n-p)-\sum_{i=0}^{n-p-1} \bar{N}^{i} \bar{B}_{w 2} w(k+i)$.

Since $\bar{N}$ is a nilpotent matrix with $\bar{N}^{n-p}=0$, we know that for $k>n-p, \bar{N}^{k}=0$. Therefore, (14) becomes $\bar{x}_{2}(k)=$ $-\sum_{i=0}^{n-p-1} \bar{N}^{i} \bar{B}_{w 2} w(k+i)$. With $w(k) \in \mathcal{W}, \forall k \in \mathbb{N}$, the set for $\bar{x}_{2}$ can be computed by $\Theta_{2}=\bigoplus_{i=0}^{n-p-1}\left(-\bar{N}^{i} \bar{B}_{w 2} \mathcal{W}\right)=\left(-\bar{B}_{w 2} \mathcal{W}\right) \oplus$ $\left(-\bar{N} \bar{B}_{w 2} \mathcal{W}\right) \oplus \cdots \oplus\left(-\bar{N}^{n-p-1} \bar{B}_{w 2} \mathcal{W}\right)$. Finally, we derive the mRPI set $\Omega^{n}=P_{1} \Theta_{1} \oplus \bar{P}_{2} \Theta_{2}$.

Remark 4. Theorem 3 builds on the assumption that the time domain of solution to the system (1) is $\mathbb{N}$. The existence of this infinite-time trajectory leads to a positive invariance property although the system is not causal. Theorem 3 should be reconsidered in case that the trajectories are defined only for a finite-time window.

For a non-causal descriptor system (1), we also present the results of the compatibility check for any initial state $x(0)$ and convergence time. The proofs are similar to the ones of Corollary 1 and Theorem 2.

Corollary 2. Consider an initial state $x(0)$ of a non-causal descriptor system (1). If $\bar{x}_{2}(0) \notin \Theta_{2}$ where $x(0)=\bar{P}_{1} \bar{x}_{1}(0)+\bar{P}_{2} \bar{x}_{2}(0)$, then $x(0)$ is compatible for (1) irrespective of any disturbance realization $w(0) \in$ $\mathcal{W}$.

Theorem 4 (Convergence Time for Non-causal Descriptor Systems). Consider a non-causal descriptor system (1) affected by disturbances $w(k) \in \mathcal{W}, \forall k \in \mathbb{N}$ and let the set $\Omega_{0}^{n} \supseteq \Omega^{n}$ with $\Omega_{0}^{n}=P_{1} \hat{\Theta}_{1,0} \oplus$ $P_{2} \Theta_{2}$. For a compatible initial state $x(0)$, the system trajectory $x(k)$ converges to $\Omega_{0}^{n}$ in $T_{c_{n}}$ iterations, that is, $\bar{x}_{1}(k)$ defined in (11) belongs to $\hat{\Theta}_{1,0}$, for $k \geq T^{c_{n}}$, where $T^{c_{n}}$ is the convergence time corresponding to (1) and depends on $x(0)$ and $\bar{\varepsilon}$. 
As an extension for a non-causal descriptor system (1), we now focus on trajectories defined only on a finite-time window, that is $x(k), k \in \mathbb{Z}_{[0, L]}$ with $L>0$. The dynamics of a non-causal descriptor system (1) obey the equivalent subsystems in (12) but the set-invariance characterization needs to be relaxed in order to consider the finite number of dynamical constraints as well as the structural particularities (algebraic equations) related to anti-causality.

The difficulties are related to a combination of causal and anti-causal dynamics in (12a) and (12b). For (12a), the positive invariance will be the appropriate concept while for (12b), the negative invariance offers the suitable framework in a predefined finite-time window $L$.

Theorem 5 (L-steps RNI Set). Consider the anti-causal subsystem (12b). A set $\Upsilon$ is L-steps RNI if

$\Upsilon \supseteq \bar{N} \Upsilon \oplus\left\{-\bar{B}_{w 2} \mathcal{W}\right\} \supseteq \cdots \supseteq \bar{N}^{L} \Upsilon \bigoplus_{i=0}^{L-1}\left\{-\bar{N}^{i} \bar{B}_{w 2} \mathcal{W}\right\}$

Proof. From (12b), we have $\bar{x}_{2}(k)=\bar{N} \bar{x}_{2}(k+1)-\bar{B}_{w 2} w(k)$. For a finite time window $L>0, \bar{x}_{2}(L) \in \Upsilon$. By the backward propagations of $\bar{x}_{2}(k+L) \in \Upsilon$ for any $k \in \mathbb{Z}_{[-L, 0]}$, we can derive (15).

Corollary 3. Given $L_{1}$ - and $L_{2}$-step RNI sets $\Upsilon_{1}$ and $\Upsilon_{2}$ with $L_{1} \geq$ $L_{2} \geq n-p$ satisfying $\Upsilon_{1} \supseteq \Upsilon_{2}$, then $\bar{N}^{l} \Upsilon_{1} \bigoplus_{i=0}^{l-1}\left\{-\bar{N}^{i} \bar{B}_{w 2} \mathcal{W}\right\} \supseteq$ $\bar{N}^{l} \Upsilon_{2} \bigoplus_{i=0}^{l-1}\left\{-\bar{N}^{i} \bar{B}_{w 2} \mathcal{W}\right\}, \forall l \geq 0$.

Proof. The relationship (15) holds for $l=0$ as $\Upsilon_{1} \supseteq \Upsilon_{2}$. Suppose $\bar{N}^{l} \Upsilon_{1} \bigoplus_{i=0}^{l-1}\left\{-\bar{N}^{i} \bar{B}_{w 2} \mathcal{W}\right\} \supseteq \bar{N}^{l} \Upsilon_{2} \bigoplus_{i=0}^{l-1}\left\{-\bar{N}^{i} \bar{B}_{w 2} \mathcal{W}\right\}$ holds for some $l \geq 0$. Then, by pre-multiplying with $\bar{N}$ and Minkowski summing the set $\left\{-\bar{B}_{w 2} \mathcal{W}\right\}$ on both sides, we obtain $\bar{N}^{l+1} \Upsilon_{1} \bigoplus_{i=0}^{l}$ $\left\{-\bar{N}^{i} \bar{B}_{w 2} \mathcal{W}\right\} \supseteq \bar{N}^{l+1} \Upsilon_{2} \bigoplus_{i=0}^{l}\left\{-\bar{N}^{i} \bar{B}_{w 2} \mathcal{W}\right\}$. The proof is completed by induction.

Remark 5. The set $\Theta_{2}$ in (13b) is $L$-steps RNI with respect to (12b), $\forall L>0$.

Remark 6. Consider the set $\Theta_{2}$ as in (13b). An L-steps RNI set with respect to (12b) can be constructed iteratively starting from $\Upsilon_{0}=\Theta_{2}$ and for $i \in \mathbb{Z}_{[1, L]}$, the recursive construction is given by

$\Upsilon_{i}=\left\{x \in \mathcal{X}_{2}: \exists w \in \mathcal{W}, \bar{N} x-\bar{B}_{w 2} w \in \Upsilon_{i-1}\right\}$,

and $\mathcal{X}_{2} \subseteq \mathbb{R}^{(n-p)}$ is a pre-defined set of state constraints for $\bar{x}_{2}$.

Theorem 6 (L-steps RNI Set of Non-causal Descriptor Systems). Consider a non-causal descriptor system (1) in with the Kronecker form in (6). The set

$\Omega=\bar{P}_{1} \Theta_{1} \oplus \bar{P}_{2} \Upsilon$,

guarantees that $x(k) \in \Omega, \forall k \in \mathbb{Z}_{[0, L]}$ if $\bar{x}_{1}(0) \in \Theta_{1}$ and $\bar{x}_{2}(L) \in \Upsilon$.

Proof. From (13a), the set $\Theta_{1}$ is RPI for the dynamics of $\bar{x}_{1}(k)$. If $\bar{x}_{1}(0) \in \Theta_{1}$, then it follows $\bar{x}_{1}(k) \in \Theta_{1}, \forall k \in \mathbb{Z}_{[0, L]}$. Meanwhile, the set $\Upsilon$ is $L$-steps RNI for $\bar{x}_{2}(k)$ as discussed in Theorem 5. If $\bar{x}_{2}(L) \in \Upsilon$, then it follows $\bar{x}_{2}(k) \in \Upsilon, \forall k \in \mathbb{Z}_{[0, L]}$. Thus, we obtain $\Omega$ by a linear mapping of $\Theta_{1}$ and $\Upsilon$ as in (17).

Proposition 1. Consider a non-causal descriptor system (1) in the restricted equivalent form (6) and define a finite-time trajectory $x(k)$ for $k \in \mathbb{Z}_{[0, L]}$ with $L>0$. If $x(0) \in \Omega_{0}$ for $L>s=n-p$ with $\bar{N}^{s}=0$ and $\bar{N}^{s-1} \neq 0$, then $x(k) \in \Omega_{0}$ for $k \in \mathbb{Z}_{[0, L-s]}$ and $x(k) \in \Omega_{k-(L-s)}$ for $k \in \mathbb{Z}_{[L-s, L]}$, where $\Omega_{i}=\bar{P}_{1} \Theta_{1} \oplus \bar{P}_{2} \Upsilon_{i}$ with $\Upsilon_{i}$ in (16).
Proof. For $k \in \mathbb{Z}_{[0, L-s]}$, from (14), $x(k)$ is contained in the RI set $\Omega_{0}=\Theta_{2}$ as defined in (17). On the other hand, for $k \in$ $\mathbb{Z}_{[L-S, L]}$, the anti-causal component is contained in $\Upsilon_{i}$, which can be propagated by using (16) leading to the confinement of the finite time trajectories for $L-s<k<L$.

\section{Active mode detection for discrete-time descriptor systems}

In this section, we propose an active mode detection mechanism based on the RPI set characterizations for systems with multiple modes of operation and no switch between different modes. The objective is the identification of the current operating mode in a finite time with any initial state $x(0)$. This operating mode will be detected from a (finite) predefined set of modes of operation. The algorithmic procedure is able to detect the current operating mode based on the offline design of active detection input and online monitoring.

\subsection{Problem formulation}

Consider a family of discrete-time descriptor systems corresponding to multiple modes of operation as

$E^{\sigma} x(k+1)=A^{\sigma} x(k)+B^{\sigma} u(k)+B_{w}^{\sigma} w(k)$,

where $E^{\sigma} \in \mathbb{R}^{n \times n}$ with $\operatorname{rank}\left(E^{\sigma}\right) \leq n, A^{\sigma} \in \mathbb{R}^{n \times n}, B^{\sigma} \in \mathbb{R}^{n \times m}$, $B_{w}^{\sigma} \in \mathbb{R}^{n \times q}$, and $\sigma \in \Sigma_{d}=\{1, \ldots, \bar{d}\}$ denotes the constant mode index and $u(k) \in \mathbb{R}^{m}$ denotes an additive input vector at time instant $k$. It is assumed that the descriptor system (18) is regular and stable for any $\sigma \in \Sigma_{d}$, then it follows that matrices $\left(E^{\sigma}-A^{\sigma}\right)$ are non-singular.

In order to check the compatibility of any state $x(k)$, based on the Kronecker canonical form in Lemma 4, let us denote the partitioning form $x=\left[x_{1}^{\top} x_{2}^{\top}\right]^{\top}$, where $x_{1} \in \mathbb{R}^{p}$ is the dynamical part corresponding to the dynamics (12a) and $x_{2} \in \mathbb{R}^{(n-p)}$ is the algebraic part corresponding to the algebraic equation (12b). Based on this notation, we also denote $B^{\sigma}=\left[\begin{array}{ll}B_{1}^{\sigma^{\top}} & B_{2}^{\sigma^{\top}}\end{array}\right]^{\top}$ and $B_{w}^{\sigma}=\left[\begin{array}{ll}B_{w_{1}}^{\sigma}{ }^{\top} & B_{w_{2}}^{\sigma}{ }^{\top}\end{array}\right]^{\top}$.

Let us also denote the transformation $\left(\bar{Q}^{i}, \bar{P}^{i}\right)$ for the descriptor system (18) at mode $i \in \Sigma_{d}$ such that $\bar{Q}^{i} E^{i} \bar{P}^{i}$ and $\bar{Q}^{i} A^{i} \bar{P}^{i}$ satisfy the Kronecker canonical form in (6). From the RPI set characterizations in Section 3 , the RPI set $\tilde{\mathcal{P}}^{i}$ composed of $\tilde{\mathcal{P}}^{i}=$ $\bar{P}_{1}^{i} \Phi_{1}^{i} \oplus \bar{P}_{2}^{i} \Phi_{2}^{i}$ with $\bar{P}^{i}=\left[\bar{P}_{1}^{i} \bar{P}_{2}^{i}\right]$.

Theorem 7. A state $x(k)=\left[x_{1}(k)^{\top} x_{2}(k)^{\top}\right]^{\top}$ is compatible with respect to the descriptor system (18) in an operating mode $i \in \Sigma_{d}$ only if $x_{2}(k)$ satisfies

$x_{2}(k) \in \bar{P}_{2}^{i} \Phi_{2}^{i}$.

Proof. Based on the Kronecker canonical form in (6), with the transformation $\left(\bar{Q}^{i}, \bar{P}^{i}\right)$ in mode $i \in \Sigma_{d}$, for a compatible state $x(k)$, the corresponding algebraic equation (12b) should be satisfied. Thus, the condition (19) could be used for checking the operating mode $i \in \Sigma_{d}$.

Based on the above theorem, we state the following corollary without proof.

Corollary 4. For an initial state $x(0)=\left[x_{1}(0)^{\top} x_{2}(0)^{\top}\right]^{\top}$, if $x_{2}(0) \notin$ $\bar{P}_{2}^{i} \Phi_{2}^{i}$, then the initial operating mode set $\Sigma_{d}(0)=\Sigma_{d} \backslash\{i\}$.

The objective of the mode detection is to decide which mode $\sigma \in \Sigma_{d}$ is active in (18) by monitoring the current state $x(k)$ and without prior knowledge on $w(k) \in \mathcal{W}$. The initial state $x(0)$ is assumed to be known and we make use of the RPI sets of (18) of each mode $\sigma \in \Sigma_{d}$ as $\tilde{\mathcal{P}}^{\sigma}$ when $u \equiv 0$. For a state $x(k)$ of (18), 


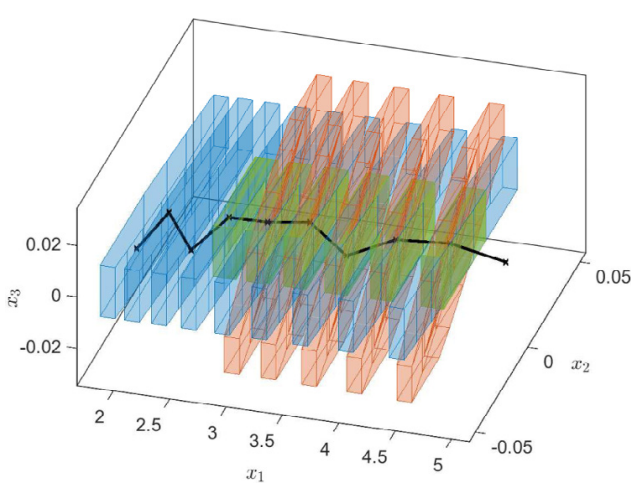

Fig. 1. A passive mode detection example . (For interpretation of the references to color in this figure legend, the reader is referred to the web version of this article.)

$\forall k \in \mathbb{N}$, in the mode $i \in \Sigma_{d}$, we split $x(k)=\bar{x}^{i}(k)+\tilde{x}^{i}(k)$ with the nominal and perturbed dynamics

$E^{i} \bar{\chi}^{i}(k+1)=A^{i} \bar{x}^{i}(k)$,

$E^{i} \tilde{x}^{i}(k+1)=A^{i} \tilde{x}^{i}(k)+B_{w}^{i} w(k)$

where $\bar{x}^{i} \in \mathbb{R}^{n}$ and $\tilde{x}^{i} \in \mathbb{R}^{n}$.

The basic passive mode detection mechanism $(u \equiv 0)$ can be summarized as follows:

Proposition 2. Consider the compatible initial state $x(0)=\bar{x}_{i}(0)+$ $\tilde{x}^{i}(0)$ satisfying $x(0)-\bar{x}^{i}(0) \in \tilde{\mathcal{P}}^{i}$, and let the set of viable modes be initialized as $\Sigma(0)=\Sigma_{d}$. Given the state measured at time $k$, if $x(k) \notin\left\{\bar{x}^{i}(k) \oplus \tilde{\mathcal{P}}^{i}\right\}$, then the mode $i$ is not the current operating mode, that is, $\Sigma(k)=\Sigma(k) \backslash\{i\}$.

Proof. The error dynamics $\tilde{x}^{i}(k)=x(k)-\bar{x}^{i}(k)$ satisfy (20b) and the initialization ensures $\tilde{x}^{i}(k) \in \tilde{\mathcal{P}}^{i}$. If the system (18) is operating in mode $i$, then the positive invariance of $\tilde{\mathcal{P}}^{i}$ is guaranteed using (20b). Whenever $x(k) \notin\left\{\bar{x}^{i}(k) \oplus \tilde{\mathcal{P}}^{i}\right\}$, the positive invariance is violated and the mode $i$ cannot represent the current operating mode.

Remark 7. Assuming $\Sigma_{d}(k)=\Sigma_{d}(k-1) \backslash\{i\}, \forall i \in \Sigma_{d}$ such that $x(k)-\bar{x}^{i}(k) \notin \tilde{\mathcal{P}}^{i}$, then Card $\left(\Sigma_{d}(k)\right)$ is monotonically decreasing as time $k$ increases. However, one cannot guarantee Card $\left(\Sigma_{d}(k)\right) \rightarrow 1$.

Example. Consider three modes of operation in (18). As shown in Fig. 1, from an initial state $x(0)$, the mode shown in blue sets is detected after several steps. As time $k$ increases, the modes in red and green sets are discarded. Note that the system state trajectory $x(k)$ may always stay in the intersection of three sets during propagations. Thus, we cannot discard any mode.

This passive mode detection does not guarantee the mode identifiability regardless of the initial conditions. Indeed, $\bigcap_{\sigma \in \Sigma_{d}}$ $\tilde{\mathcal{P}}^{\sigma} \neq \varnothing$ and thus there exists at least a realization $w(k), \forall k \in \mathbb{N}$, which does not allow to decrease the cardinality of $\Sigma_{d}(k)$ and eventually identify the current mode of operation. The active mode detection is intended to enhance the monitoring process by the injection of an excitation signal.

\subsection{Design of active detection input}

For any two different modes $i, j \in \Sigma_{d}$, the active detection input denoted by $u(k)$ is designed to guarantee $\mathcal{P}^{i}(k) \cap \mathcal{P}^{j}(k)=$ $\varnothing$ for some $k \in \mathbb{N}$, where $\mathcal{P}^{i}(k)$ and $\mathcal{P}^{j}(k)$ denote the tube of trajectories parameterized by $u(k)$. From (18), the system (18) in modes $i$ and $j$ can be formulated as

$E^{i} x(k+1)=A^{i} x(k)+B^{i} u(k)+B_{w}^{i} w(k)$,

$E^{j} x(k+1)=A^{j} x(k)+B^{j} u(k)+B_{w}^{j} w(k)$.

Recall that for $u(k)=0$ in $(21)$, it follows $\mathcal{P}^{i}(k)=\tilde{\mathcal{P}}^{i}$ and $\mathcal{P}^{j}(k)=\tilde{\mathcal{P}}^{j}$.

Similar to (20), assuming the system (18) in mode $i \in \Sigma_{d}$, we split $x(k)=\bar{x}^{i}(k)+\tilde{x}^{i}(k)$ with

$E^{i} \bar{x}^{i}(k+1)=A^{i} \bar{x}^{i}(k)+B^{i} u(k)$,

$E^{i} \tilde{x}^{i}(k+1)=A^{i} \tilde{x}^{i}(k)+B_{w}^{i} w(k)$.

With an active detection input $u(k), \forall k \in \mathbb{N}$, the state $x(0)$ has to be decomposed as $x(0)=\bar{x}^{i}(0)+\tilde{x}^{i}(0)$ (for instance in mode $i \in \Sigma_{d}$ ) to satisfy the algebraic equations in the descriptor model (18). Based on this observation, we introduce the following proposition to check whether the initial state $x(0)$ is compatible by testing the satisfaction of algebraic equations in (18) for different modes.

Proposition 3. Given the set of modes $\Sigma_{d}$. For any $i \in \Sigma_{d}$ such that $\operatorname{rank}\left(E^{i}\right)<n$, if $B_{2}^{i} \neq 0$, then $\exists u(0)$ such that

$x(0) \notin \tilde{\mathcal{P}}^{i}$.

Proof. From (22b), we know $x(0)=\bar{x}^{i}(0)+\tilde{x}^{i}(0)$ and $\tilde{x}^{i}(0) \in \tilde{\mathcal{P}}^{i}$. Based on the nominal descriptor dynamics $(22 a), \bar{x}^{i}(0)$ is also constrained by $u(0)$ at time $k=0$. If $B_{2}^{i} \neq 0$, then $\bar{x}^{i}(0) \neq 0$. Considering the boundedness of $\tilde{\mathcal{P}}^{i}$ and the fact that $x(0)=\bar{x}^{i}(0)+$ $\tilde{x}^{i}(0)$, there exists $u(0)$ acting on $\bar{x}^{i}(0)$ that satisfies (23).

The result in Proposition 3 shows that descriptor systems have structural advantages in view of mode detection, that is, the algebraic equations in a descriptor systems must hold. When an additional detection input signal is applied, by checking (23), some modes can be discarded.

We now present the procedure to design a constant active detection input $\bar{u} \neq 0$ that can be applied to the system (18) with a finite detection time $N_{T}$ as

$u(k)= \begin{cases}\bar{u}, & \text { if } k \leq N_{T}-1, \\ 0, & \text { otherwise. }\end{cases}$

With this constant input $\bar{u}$, (22) becomes

$E^{i} \bar{x}^{i}(k+1)=A^{i} \bar{x}^{i}(k)+B^{i} \bar{u}$,

$E^{i} \tilde{x}^{i}(k+1)=A^{i} \tilde{x}^{i}(k)+B_{w}^{i} w(k)$.

Recall $x(0)=\left[x_{1}(0)^{\top}, x_{2}(0)^{\top}\right]^{\top}$. The initial condition is given by $\bar{x}_{1}^{i}(0)=x_{2}(0)$ and $\bar{x}_{2}^{i}(0)$ satisfies (24a) with $\bar{u}$.

By definition of the RPI set, we denote $\tilde{x}^{i}(k+1) \in \tilde{\mathcal{P}}^{i}, \forall \tilde{x}^{i}(k) \in$ $\tilde{\mathcal{P}}^{i}, \forall w(k) \in \mathcal{W}, \forall k \in \mathbb{N}$. The system trajectory in mode $i$ belongs to the parameterized RPI set, that is, $x(k) \in \mathcal{P}^{i}(k)=\left\{\bar{x}^{i}(k) \oplus \tilde{\mathcal{P}}^{i}\right\}$, with $\bar{x}^{i}(k)$ obtained from (24a) and $\forall w(k) \in \mathcal{W}, \forall k \in \mathbb{N}$.

From the nominal dynamics (24a), the stability is guaranteed when the system evolves towards the equilibrium point

$\bar{x}_{\infty}^{i}=\left(E^{i}-A^{i}\right)^{-1} B^{i} \bar{u}$.

In the following theorem, we present the set separation condition for the design of $\bar{u}$.

Theorem 8. For any two modes $i, j \in \Sigma_{d}$, the sets

$\mathcal{P}_{\infty}^{i}=\left\{\bar{x}_{\infty}^{i} \oplus \tilde{\mathcal{P}}^{i}\right\}, \mathcal{P}_{\infty}^{j}=\left\{\bar{x}_{\infty}^{j} \oplus \tilde{\mathcal{P}}^{j}\right\}$ 


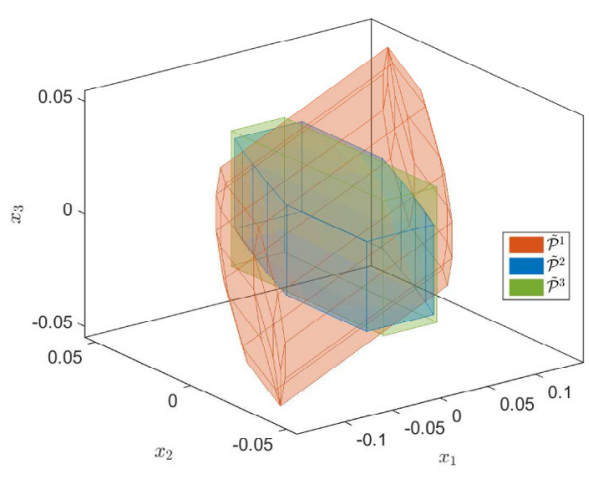

(a) Three mRPI sets

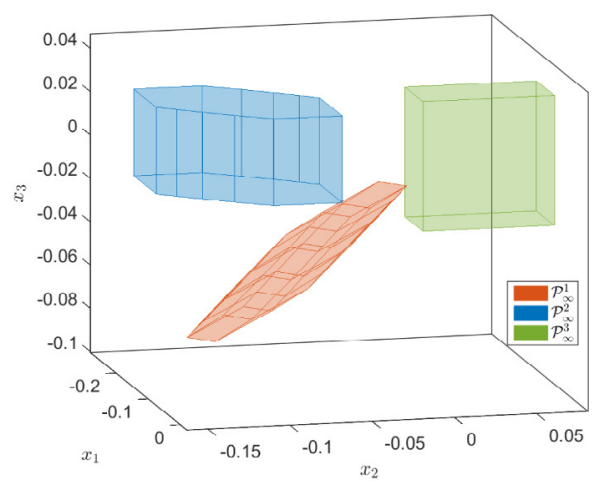

(b) Seperated mRPI sets

Fig. 2. Three mRPI sets and separated sets.

satisfy $\mathcal{P}_{\infty}^{i} \cap \mathcal{P}_{\infty}^{j}=\varnothing$ if and only if there exists an active detection input $\bar{u}$ such that

$\left(\left(E^{i}-A^{i}\right)^{-1} B^{i}-\left(E^{j}-A^{j}\right)^{-1} B^{j}\right) \bar{u} \notin \mathcal{S}^{i j}$.

Proof. From (26), $\mathcal{P}_{\infty}^{i} \cap \mathcal{P}_{\infty}^{j}=\varnothing$ is equivalent to

$$
\left\{\bar{x}_{\infty}^{i} \oplus \tilde{\mathcal{P}}^{i}\right\} \cap\left\{\bar{x}_{\infty}^{j} \oplus \tilde{\mathcal{P}}^{j}\right\}=\varnothing
$$

By adding $-\bar{x}_{j}^{\infty}$ to the above both sets in (28), we obtain $\left\{\bar{x}_{\infty}^{i} \oplus\left(-\bar{x}_{\infty}^{j}\right) \oplus \tilde{\mathcal{P}}^{i}\right\} \cap\left\{\bar{x}_{\infty}^{j} \oplus\left(-\bar{x}_{\infty}^{j}\right) \oplus \tilde{\mathcal{P}}^{j}\right\}=\varnothing$, which can be simplified as $\left\{\left(\bar{x}_{\infty}^{i}-\bar{x}_{\infty}^{j}\right) \oplus \tilde{\mathcal{P}}^{i}\right\} \cap \tilde{\mathcal{P}}^{j}=\varnothing$ leading to (27) based on (25).

Let us denote the half-space representation of the set $\mathcal{S}_{i j}$ as $\mathcal{S}_{i j}=\left\{x \in \mathbb{R}^{n}: H_{i j} x \leq b_{i j}\right\}, \forall i, j \in \Sigma_{d}$,

where $H_{i j} \in \mathbb{R}^{p_{i j} \times n}, b_{i j} \in \mathbb{R}^{p_{i j}}$, and $p_{i j}$ is the total number of the linear constraints corresponding to $\mathcal{S}_{i j}$.

Based on the set separation condition in (27), the constant active detection input $u \in\left[u_{\min }, u_{\max }\right]$ can be obtained by solving offline the following mixed-integer optimization problem.

Problem 1 (Constant Active Detection Input).

$$
\text { minimize } u^{2} \text {, }
$$

subject to $\bar{x}_{\infty}^{i}=\left(E^{i}-A^{i}\right)^{-1} B^{i} u$,

$\bar{x}_{\infty}^{j}=\left(E^{j}-A^{j}\right)^{-1} B^{j} u$,

$u_{\min } \leq u \leq u_{\max }$

$H_{i j}\left(\bar{x}_{\infty}^{i}-\bar{x}_{\infty}^{j}\right) \geq b_{i j}-M_{r} \Delta_{i j}+\epsilon_{r}$,

$\Delta_{i j}=\left\{\delta_{1}, \ldots, \delta_{p_{i j}}\right\} \in\{0,1\}$,

$$
\sum_{l=1}^{p_{i j}} \delta_{l}=p_{i j}-1, \forall i, j \in \Sigma_{d}, i \neq j
$$

with an arbitrary large positive scalar $M_{r}$ and an arbitrary small positive scalar $\epsilon_{r}$.

The optimal solution of Problem 1 defines the constant active detection input $\bar{u}=u$.

Remark 8. The computational complexity of Problem 1 relies on the total amount of decision variables that includes the vector $u \in \mathbb{R}^{m}$ and the binary variables in $\Delta_{i j}$. The worst-case complexity of $\Delta_{i j}$ is $2^{p_{i j}}$, where $p_{i j}$ is the number of linear constraints for the set $S_{i j}$.
Example. Consider the same three modes of operation in (18). By solving Problem 1 , a constant active detection input $\bar{u}$ can be obtained. As shown in Fig. 2, mRPI sets for three modes can be separated by $\bar{u}$.

With the constant detection input obtained from solving Problem 1, the guaranteed mode detection result is presented in the following theorem.

Theorem 9. If $\bar{u}$ is a feasible solution of Problem 1, then for any initial state $x(0)$, there exists a finite time $N_{T}(x(0))$ such that the detection $\operatorname{Card}\left(\Sigma_{d}(k)\right)=1$ is achieved in $k \leq N_{T}(x(0))$. Moreover, the convergence time from $x(0)$ to the set $\mathcal{P}_{\infty}^{i}$ denoted as $T_{c}^{i}$ can be computed explicitly for any $i \in \Sigma_{d}$. Then, the upper bound for the detection time is

$N_{T}(x(0))=\max _{i} T_{c}^{i}$.

Proof. By the design of $\bar{u}$, it is guaranteed $\mathcal{P}_{\infty}^{i} \cap \mathcal{P}_{\infty}^{j}=\varnothing$ for any two modes $i, j \in \Sigma_{d}$. For a given initial state $x(0)$ compatible with the mode $i$ in (24), one has $x\left(T_{c}^{i}\right) \in \mathcal{P}_{\infty}^{i}$ independent of the operating mode. But $\mathcal{P}_{\infty}^{i} \cap \mathcal{P}_{\infty}^{j}=\varnothing$ for all $i \neq j$ and $x\left(N_{T}(x(0))\right) \in$ $\mathcal{P}_{\infty}^{i}$ only holds for the current operating mode.

\subsection{Active mode detection algorithm}

Based on the above results, we now propose an algorithm to achieve the mode detection by updating online the active input according to the monitoring of the compatible modes. Overall, this leads to a piecewise constant signal and a detection time upper-bounded by $N_{T}(x(0))$.

Offline procedure: For any $\Sigma \subseteq \Sigma_{d}$ with $\operatorname{Card}(\Sigma) \geq 2$, compute $\bar{u}_{\Sigma}$ as the solution of Problem 1 .

Online procedure: Input an initial state $x(0)$;

(i) Initialize $\Sigma_{d}(0)=\Sigma_{d}$;

(ii) Compute the compatible state $\bar{x}^{i}(0)$ with $u(0)=\bar{u}_{\Sigma_{d}(0)}$ and $x(0)$;

(iii) $k=0$;

(iv) while $\left(\operatorname{Card}\left(\Sigma_{d}(k)\right)>1\right)$

(1) for $i \in \Sigma_{d}(k)$, if $x(k) \notin\left\{\bar{x}^{i}(k) \oplus \tilde{\mathcal{P}}^{i}\right\}$, then $\Sigma_{d}(k)=$ $\Sigma_{d}(k) \backslash\{i\} ;$

(2) $u(k)=\bar{u}_{\Sigma_{d}(k)}$;

(3) Update the nominal state $\bar{x}^{i}(k+1)$ by (24a);

(4) $k=k+1$;

(v) Obtain $\operatorname{Card}\left(\Sigma_{d}(k)\right)=1$ and the operating mode is detected. 


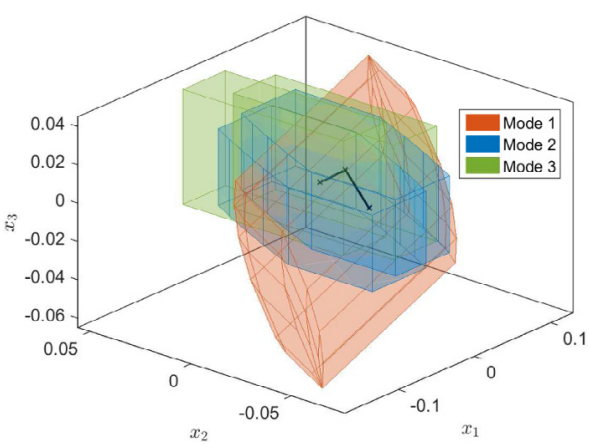

Fig. 3. Active model detection with $\bar{u}$. (For interpretation of the references to color in this figure legend, the reader is referred to the web version of this article.)

Example. By applying the above algorithm to the same example, the simulation result is shown in Fig. 3. From an initial state $x(0)$, the operating mode can be detected at time $k=3$ and the system (18) is in the Mode 2 since the state trajectory only stays in the blue set at time $k=3$.

Remark 9. Active mode detection can also achieved via online redesign of the separation signals. A receding sequence of detection inputs can be designed with a given detection window through a corresponding mixed-integer optimization structurally similar to Problem 1. Then, online monitoring procedure can be carried out with these time-varying detection inputs. As a result, the operating mode can be detected within a predefined window.

\section{Conclusion}

In this paper, we have studied robust invariant set characterizations of discrete-time descriptor systems in both causal and non-causal cases. Based on two restricted equivalent forms of descriptor systems, the explicit results on robust invariant set characterizations are provided. Besides, we have also proposed an active mode detection mechanism based on RPI set-invariance characterizations for discrete-time descriptor system with multiple modes of operation. Based on the separation of RPI sets of descriptor systems, we have proposed a method to design an active detection input and an active mode detection algorithm.

\section{Acknowledgments}

This work was partially funded by the Spanish State Research Agency (AEI) and the European Regional Development Fund (ERFD) through the project DEOCS (ref. DPI2016-76493C3-3-R), the FPI grant (ref. BES-2014-068319), the FPI mobility grants (ref. EEBB-I-17-12257 and EEBB-I-18-12900), and by a grant of Ministry of Research and Innovation, CNCS - UEFISCDI, Romania project number PN-III-P1-1.1-TE-2016-0862, MOSCBIOS, within PNCDI III.

\section{References}

Alamo, T., Cepeda, A., Fiacchini, M., \& Camacho, E. (2009). Convex invariant sets for discrete-time lur'e systems. Automatica, 45(4), 1066-1071.

Biegler, L., Campbell, S., \& Mehrmann, V. (2012). Control and optimization with differential-algebraic constraints. Society for Industrial and Applied Mathematics.

Blanchini, F. (1999). Set invariance in control. Automatica, 35(11), 1747-1767.

Blanchini, F., Casagrande, D., Giordano, G., Miani, S., Olaru, S., \& Reppa, V. (2017). Active fault isolation: a duality-based approach via convex programming. SIAM Journal on Control and Optimization, 55(3), 1619-1640.

Dai, L. (1989). Singular control systems. Springer.
Duan, G. (2010). Analysis and design of descriptor linear systems. Springer.

Gerdin, M. (2004). Computation of a canonical form for linear differentialalgebraic equations. Technical report, Linköping University, http://www control.isy.liu.se/research/reports/2004/2602.pdf.

Halanay, A., \& Rasvan, V. (2000). Stability and stable oscillations in discrete time systems. CRC Press.

Kofman, E., Haimovich, H., \& Seron, M. (2007). A systematic method to obtain ultimate bounds for perturbed systems. International Journal of Control, 80(2), $167-178$

Kolmanovsky, I., \& Gilbert, E. (1998). Theory and computation of disturbance invariant sets for discrete-time linear systems. Mathematical Problems in Engineering, 4(4), 317-367.

Marseglia, G., \& Raimondo, D. (2017). Active fault diagnosis: A multi-parametric approach. Automatica, 79, 223-230.

Mayne, D., Seron, M., \& Raković, S. (2005). Robust model predictive control of constrained linear systems with bounded disturbances. Automatica, 41(2), 219-224.

Oară, C., \& Andrei, R. (2013). Numerical solution to a descriptor discrete-time algebraic Riccati equation. Systems E' Control Letters, 62(2), 201-208.

Olaru, S., De Doná, J., Seron, M., \& Stoican, F. (2010). Positive invariant sets for fault tolerant multisensor control schemes. International Journal of Control, 83(12), 2622-2640.

Raimondo, D., Marseglia, G., Braatz, R., \& Scott, J. (2016). Closed-loop input design for guaranteed fault diagnosis using set-valued observers. Automatica, 74, 107-117.

Raković, S., Kerrigan, E., Kouramas, K., \& Mayne, D. (2005). Invariant approximations of the minimal robust positively invariant set. IEEE Transactions on Automatic Control, 50(3), 406-410.

Riaza, R. (2008). Differential-algebraic systems: Analytical aspects and circuit applications. World Scientific Publishing Company.

Seron, M., \& De Doná, J. (2015). On robust stability and set invariance of switched linear parameter varying systems. International Journal of Control, 88(12), 2588-2597.

Seron, M., De Doná, J., \& Olaru, S. (2012). Fault tolerant control allowing sensor healthy-to-faulty and faulty-to-healthy transitions. IEEE Transactions on Automatic Control, 57(7), 1657-1669.

Stoican, F., \& Olaru, S. (2013). Set-theoretic fault-tolerant control in multisensor systems. Wiley-ISTE.

Varga, A. (2017). Solving fault diagnosis problems. Springer.

Wang, Y., Olaru, S., Valmorbida, G., Puig, V., \& Cembrano, G. (2017). Robust invariant sets and active mode detection for discrete-time uncertain descriptor systems. In 56th IEEE conference on decision and control (pp. 5648-5653).

Wang, Y., Puig, V., \& Cembrano, G. (2017). Non-linear economic model predictive control of water distribution networks. Journal of Process Control, 56, 23-34

Xu, F., Puig, V., Ocampo-Martinez, C., Stoican, F., \& Olaru, S. (2014). Actuator-fault detection and isolation based on set-theoretic approaches. Journal of Process Control, 24(6), 947-956. 